UPR-522T

\title{
STRINGY INSTANTONS
}

\author{
RAM BRUSTEIN and BURT A. OVRUT \\ Department of Physics \\ University of Pennsylvania \\ Philadelphia, PA 19104
}

\begin{abstract}
A canonical Lorentz invariant field theory extension of collective field theory of $d=1$ matrix models is presented. We show that the low density, discrete, sector of collective field theory includes single eigenvalue Euclidean instantons which tunnel between different vacua of the extended theory. These "stringy" instantons induce non-perturbative effective operators of strength $e^{-1 / g}$. The relationship of the world sheet description of string theory and Liouville theory to the effective space-time theory is explained.
\end{abstract}

Non-perturbative aspects of string theory are an essential piece of information needed to confront string theory predictions and the real observable world.

Matrix models, and especially $d=1$ matrix models [1], offer a unique opportunity to obtain some insight into non-perturbative string theory. The $d=1$ matrix model is the most complicated matrix model which can be explicitly solved. On the other hand, it describes the simplest space-time dynamics which is still interesting. In the double scaling limit, the $d=1$ matrix model describes strings propagating in one time dimension and one spatial dimension. An equivalent description is given in terms of a bosonic collective field theory in $1+1$ dimensions of one massless field [2], [3]. A notable feature of collective field theory is that the kinetic energy is not in canonical form and it is not Lorentz invariant. The $d=1$ matrix models, or the equivalent field theories have the power to describe nonperturbative phenomena in the associated $1+1$ string theories. By studying the generic

email: ramy@mohlsun.physics.upenn.edu

email: ovrut@penndrls.upenn.edu 
features of non-perturbative behaviour in $1+1$ dimensional string theories, as we do in this letter, one may learn about more realistic 4-dimensional string theories.

Large order growth of perturbative amplitudes is a common feature of matrix models and more complicated string theories [4]. For a review of large order behaviour of matrix model amplitudes see ref.[5]. All matrix models, as well as the critical bosonic string theory in 26 dimensions, exhibit a strange phenomenon. The magnitude of $G^{\prime}$ th order amplitudes in perturbation theory grow like $(2 G)$ !.

In the same way as $G$ ! behaviour corresponds to well understood $e^{-\frac{1}{g^{2}}}$ non-perturbative effects in quantum field theory, in matrix models the large order $(2 G)$ ! behaviour would correspond to peculiar non-perturbative effects of strength $e^{-\frac{1}{g}}$. How do these peculiar effects arise? It turns out that in matrix models, there is a new type of instanton, involving a single eigenvalue, that is responsible for these effects. For a discussion of one eigenvalue instantons see [4], [5]. They were also discussed in ref. [6] and in the context of supersymmetric matrix models in refs. [7] and [8]. We show that in matrix models, the associated effective field theory action does not obey the usual field theoretic scaling argument, $S(\phi, g) \neq \frac{1}{g^{2}} \widetilde{S}(\widetilde{\phi})$. Instead, one finds that $g$ cannot be completely scaled out of $\widetilde{S}$ due to "scale breaking terms". That is $S(\phi, g)=\frac{1}{g^{2}} \widetilde{S}(\widetilde{\phi}, g)$. It follows that a non-trivial solution can be a function of $g$. Furthermore, if for such a solution $\widetilde{S} \sim g$, then $S \sim \frac{1}{g}$. This is exactly what happens for one eigenvalue instantons.

In this letter we discuss the high and low density limits of collective field theory. We show that, in Euclidean space, there is a single eigenvalue instanton solution of the equations of motion which has action $\frac{\pi}{g}$. We then extend the collective field theory, which is a noncanonical, non-Lorentz invariant theory of a single field $\phi$, to a canonical, Lorentz invariant effective field theory of two fields $\zeta$ and $D$. This theory exhibits the scale breaking terms responsible for the unusual action, $S \sim \frac{1}{g}$, of the instantons. We show that the vacuum solutions of the effective field theory includes wormhole-like configurations. These configurations look like two identical Liouville vacua linked by a single eigenvalue instanton. The normalized action of these configurations is $S=\frac{\pi}{g}$. We then show that these configurations induce calculable operators in the $\zeta, D$ effective theory with strength $e^{-\frac{\pi}{g}}$, as conjectured by Shenker. We also review and explain the relationship of these double scaled matrix models to $1+1$ dimensional string theories, and argue that these string theories must include the new vacuum configurations, single eigenvalue instantons, and induced operators of strength $e^{-\frac{\pi}{g}}$. An extended and detailed discussion of all the issues in this letter and more can be found in [9]. 
A useful step on the road from the matrix model to string theory is collective field theory [2]. Relevant references are [10]-[12]. The idea is to start from the matrix model and, by performing a series of changes of variables, arrive at a field theory representation of the matrix model. The collective field is $\phi(x, t)=\sum_{i} \delta\left(x-\lambda_{i}(t)\right)$ where $\lambda_{i}, i=1, \ldots, N$ are the eigenvalues of the matrix. The partition function in terms of the collective field is

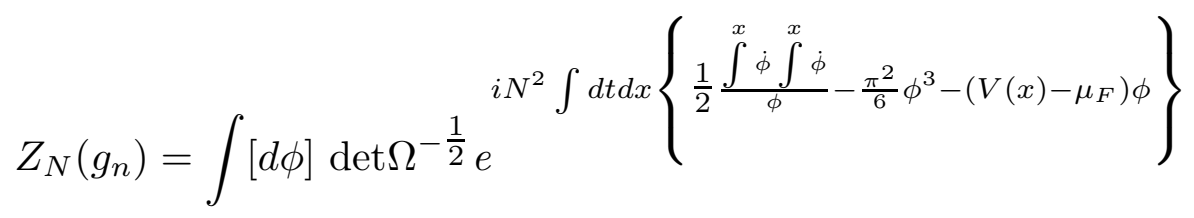

Here contact terms are omitted. Note the appearance of the factor $\operatorname{det} \Omega^{-\frac{1}{2}}$ which comes from doing the Gaussian integral over the conjugate momentum $\Pi_{\phi}$. The eigenvalues $\lambda_{i}$ are independent variables, However, for finite $N$ the collective field $\phi(x, t)$ is highly constrained. It does not correspond to an infinite number of degrees of freedom. Hence, the $\phi$ equation of motion is not obtained by varying the action with respect to $\phi$. The correct procedure is the following. Start with the equations of motion for the eigenvalues, then by using the relation between $\phi$ and $\lambda_{i}$ convert them to an equation for $\phi$. The result is

$$
\left.\frac{\partial}{\partial y} \frac{\delta S_{\mathrm{eff}}[\dot{\phi}, \phi]}{\delta \phi(y, t)}\right|_{y=\lambda_{j}(t)}=0 \quad j=1, \ldots, N
$$

Note that there are $N$ equations of motion since $y$ must be evaluated at $\lambda_{j}$ for all $j=$ $1, \ldots, N$. Furthermore, note that $\phi^{\prime} s$ satisfying the naive $\phi$ equation of motion $\frac{\delta S_{\text {eff }}[\dot{\phi}, \phi]}{\delta \phi(y, t)}=0$ also satisfy Eq.(2). However there are solutions of Eq.(2) which do not satisfy the naive equation of motion.

Consider now the limit $N \rightarrow \infty$. For our purposes it is general enough to consider a

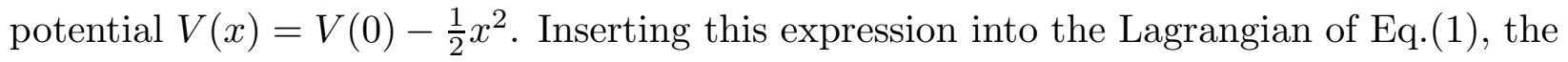
combination $V(0)-\mu_{F}$ appears. We denote $V(0)-\mu_{F}$ by $\frac{1}{2} \mu$ and assume that $\mu>0$. The double scaling limit is defined by specifying the $N$ dependence of $\mu, N \mu=\frac{1}{g}$, so that $g$ remains finite as $N \rightarrow \infty$. The classical equations of motion derived using Eq.(2) are

$$
\frac{\partial}{\partial x}\left(\int^{x} d y \partial_{t} \frac{\int^{y} \dot{\phi}}{\phi}-\frac{1}{2} \frac{\int^{x} \dot{\phi} \int^{x} \dot{\phi}}{\phi^{2}}-\frac{\pi^{2}}{2} \phi^{2}-\frac{1}{2}\left(\frac{1}{g}-x^{2}\right)\right) \mid x=\lambda_{i}(t)=0
$$

where the index $i$ now runs over $i=1, \ldots, N \rightarrow \infty$.

The high density (HD) limit of collective field theory is defined as follows. Consider a region of $x$, denoted $I$, of length $l(I)$. The number of eigenvalues in this region is $N(I)$. 
Then take the double scaling limit in such a way that $\frac{N(I)}{l(I)} \rightarrow \infty$. It is clear that in this region of $x$ there are an infinite number of eigenvalues. The collective field $\phi$ now has an infinite number of degrees of freedom and the classical equations of motion in this region are Eq.(3) without the restriction $x=\lambda_{i}(t)$. The static solution of these equations is $\phi_{0}=\frac{1}{\pi} \sqrt{x^{2}-\frac{1}{g}}$, where $|x| \geq \sqrt{\frac{1}{g}}$. Note that $\phi_{0}$ actually makes the term inside the parenthesis in Eq.(3) vanish. That is, $\phi_{0}$ is a solution of the conventional field theory equations of motion. The coupling parameter of collective field theory is $\frac{1}{\pi^{\frac{3}{2}} \phi_{0}^{2}(x)}$. However, $\frac{1}{\phi_{0}^{2}(x)}$ becomes large exactly where the classical solution $\phi_{0}$ becomes small, which is where the HD expansion breaks down. The field theory becomes strongly coupled or in other words the semi-classical expansion breaks down. What happens if this fact is ignored? Perturbation series takes its revenge by growing too fast. In fact, the $G^{\prime}$ th order in perturbation expansion grows like $(2 G)$ !. We take the HD region to be the region in $\mathrm{x}$ space where the static solution $\phi_{0}$ is defined; that is $|x| \geq \sqrt{\frac{1}{g}}$.

The low density (LD) limit of collective field theory is defined as follows. Consider a region of $x$, denoted $J$, of length $l(J)$. The number of eigenvalues in this region is $N(J)$. Then take the double scaling limit in such a way that $\frac{N(J)}{l(J)} \rightarrow$ finite. It is clear that in this region of $x$ there are a finite number of eigenvalues. The collective field $\phi$ now has a finite number of degrees of freedom in that region and the classical equations of motion are Eq.(3) where the index $i$ now runs over $i=1, \ldots, N(J)$.

In this letter, we are particularly concerned with LD regions containing a single eigenvalue, $\lambda^{*}$, in Euclidean space. Going to Euclidean time, $\theta$, and using the relation $\phi(x, \theta)=\delta(x-$ $\lambda^{*}(\theta)$ ) the equation of motion (including the contact terms) simply becomes $\frac{d^{2} \lambda^{*}}{d \theta^{2}}=-\lambda^{*}(\theta)$. The general solution of this equation is given by $\lambda^{*}(\theta)=A^{*} \cos \theta+B^{*} \sin \theta$ where $A^{*}$ and $B^{*}$ are real constants. The Euclidean LD region is taken to be $-\sqrt{\frac{1}{g}} \leq x \leq \sqrt{\frac{1}{g}}$.

As an example consider the collective field corresponding to a single eigenvalue $\lambda^{*}$ with the boundary conditions $\lambda^{*}\left(\theta_{0}\right)=\sqrt{\frac{1}{g}}$ and $\dot{\lambda}^{*}\left(\theta_{0}\right)=0$. It follows that $\lambda^{*}(\theta)=\sqrt{\frac{1}{g}} \cos \left(\theta-\theta_{0}\right)$. The corresponding collective field configuration is

$$
\phi_{\text {inst }}(x, \theta)=\delta\left(x-\frac{1}{\sqrt{g}} \cos \left(\theta-\theta_{0}\right)\right)
$$

This is an instanton that corresponds to tunneling of one eigenvalue across the barrier from $x=\sqrt{\frac{1}{g}}$ at $\theta=\theta_{0}$ to $x=-\sqrt{\frac{1}{g}}$ at $\theta=\theta_{0}+\pi$. Note that the classical solution in Eq.(四) is not a solution of the Euclidean continuation of the unconstrained field theory equations of motion. 
The action of the instanton $\phi_{\text {inst }}$ can be computed from the Euclidean continuation of (11). The result is

$$
S_{\text {eff }}\left[\phi_{\text {inst }}, \dot{\phi}_{\text {inst }}\right]=\int_{0}^{\pi} d \theta \int_{+\sqrt{\frac{1}{g}}}^{-\sqrt{\frac{1}{g}}} d x \delta\left(x-\frac{1}{\sqrt{g}} \cos (\theta)\right)\left\{\frac{1}{2 g} \sin ^{2}(\theta)-\frac{1}{2 g} \cos ^{2}(\theta)+\frac{1}{g}\right\}=\frac{\pi}{g}
$$

in agreement with the large order behavior of the perturbation series in $g$.

We begin the discussion of the effective field theory in the HD region $x \geq \sqrt{\frac{1}{g}}$. The first step is to shift the double scaled collective field $\phi$ by the classical static solution $\phi_{0}, \phi=$ $\phi_{0}+\frac{1}{\sqrt{\pi}} \partial_{x} \zeta$. To obtain a canonical kinetic term for the field $\zeta$, change coordinates to the Liouville coordinate defined by $\tau-\tau_{0}^{I}=\frac{1}{\pi} \int_{x_{0}}^{x} \frac{d y}{\phi_{0}}=\ln \left[x+\sqrt{x^{2}-\frac{1}{g}}\right]-\ln \left[x_{0}+\sqrt{x_{0}^{2}-\frac{1}{g}}\right]$. For simplicity, we take $x_{0}=\sqrt{\frac{1}{g}}$ and $\tau_{0}^{I}=\ln \sqrt{\frac{1}{g}}$. In this case $x=\sqrt{\frac{1}{g}} \cosh \left(\tau-\ln \sqrt{\frac{1}{g}}\right)$. and the range of the two coordinates is $\sqrt{\frac{1}{g}} \leq x \leq \infty$ and $\ln \left[\sqrt{\frac{1}{g}}\right] \leq \tau \leq \infty$. The static solution in the new coordinate becomes $\phi_{0}(\tau)=\frac{1}{\pi \sqrt{g}} \sinh \left(\tau-\ln \sqrt{\frac{1}{g}}\right)$. Rewritten in terms of the Liouville coordinate the classical Lagrangian is $L=L_{\zeta}\left(\zeta, \phi_{0}\right)+L_{0}\left(\phi_{0}\right)$ where $L_{\zeta}$ is given by

$$
L_{\zeta}=\int d \tau\left\{\frac{1}{2} \frac{\dot{\zeta}^{2}}{1+\frac{1}{\pi^{\frac{3}{2}} \phi_{0}^{2}(\tau)} \partial_{\tau} \zeta}-\frac{1}{2}\left(\partial_{\tau} \zeta\right)^{2}-\frac{1}{6} \frac{1}{\pi^{\frac{3}{2}} \phi_{0}^{2}(\tau)}\left(\partial_{\tau} \zeta\right)^{3}\right\}
$$

and the pure $\phi_{0}$ Lagrangian, $L_{0}$, turns into $L_{0}=\int d \tau \frac{\pi^{3}}{3} \phi_{0}^{4}$. From the cubic interaction term in $L_{\zeta}$, it follows that the coupling parameter of collective field theory is $\frac{1}{\pi^{\frac{3}{2}} \phi_{0}^{2}(\tau)}=$ $4 \sqrt{\pi} \frac{e^{-2 \tau}}{\left(1-\frac{1}{g} e^{-2 \tau}\right)^{2}}$. The coupling parameter vanishes as $\tau \rightarrow \infty$ and explodes at $\tau=\ln \left[\sqrt{\frac{1}{g}}\right]$. The low density version of collective field theory is obtained from Eq.(1) by setting $\phi=\partial_{x} \zeta$. The resulting Lagrangian is

$$
L_{\zeta}=\int d x\left\{\frac{\dot{\zeta}^{2}}{\partial_{x} \zeta}-\frac{\pi^{2}}{6}\left(\partial_{x} \zeta\right)^{3}-\frac{1}{2}\left(\frac{1}{g}-x^{2}\right) \partial_{x} \zeta\right\}
$$

We now describe a field theory that reduces to the collective field theory in the region $\tau \geq \ln \sqrt{\frac{1}{g}}$ when the various fields obtain their expectation values. The idea was discussed for $\mu=0$ in [13]. We note that the $\zeta$ theory is not Lorentz invariant. Our interpretation is that this is really a Lorentz invariant field theory of two fields, $\zeta$ and $D$, expanded around the vacuum expectation values of the two fields. The new field $D$ has a vacuum 
expectation value that breaks Lorentz invariance, and that is the reason that the $\zeta$ theory alone is not Lorentz invariant.

To find out the background independent field theory we have to identify the expectation value of the $\zeta$ and $D$ fields first. Motivated by the comparison between collective field theory and the Polyakov description of the related string theory we postulate that

$$
<G_{\mu \nu}>=\eta_{\mu \nu}, \quad<D>=-2 \tau, \quad<\zeta>=\frac{1}{g}
$$

Here we added the expectation value of the metric as well. Note that the field $D$ has the non-translation invariant vacuum expectation value.

We list the background independent form of the different quantities. $\pi^{\frac{3}{2}} \phi_{0}^{2}(\tau) \rightarrow$ $\frac{1}{4 \sqrt{\pi}} e^{-D}\left(1-\frac{1}{g} e^{D}\right)^{2}, \partial_{\tau} \zeta \rightarrow \frac{1}{2} \nabla D \cdot \nabla \zeta, \dot{\zeta}^{2}-\left(\partial_{\tau} \zeta\right)^{2} \rightarrow \nabla \zeta \cdot \nabla \zeta$. The Lorentz non-invariant quantities on the left are obtained from the Lorentz invariant quantities on the right by letting $D=<D>$ and $\zeta=<\zeta>+\zeta^{\prime}$. Also, let $\int d t d \tau=\int d^{2} x$. We can now write the $D, \zeta$ action using the previous dictionary of expressions. The result is

$$
\begin{aligned}
\mathcal{S}=\int d^{2} x & \left\{\frac{1}{2} \frac{\nabla \zeta \cdot \nabla \zeta}{1+2 \sqrt{\pi} \frac{e^{D}}{\left(1-\frac{1}{g} e^{D}\right)^{2}} \nabla \zeta \cdot \nabla D}-\frac{\sqrt{\pi}}{4} \frac{e^{D}}{\left(1-\frac{1}{g} e^{D}\right)^{2}} \frac{(\nabla \zeta \cdot \nabla D)^{3}}{1+2 \sqrt{\pi} \frac{e^{D}}{\left(1-\frac{1}{g} e^{D}\right)^{2}} \nabla \zeta \cdot \nabla D}\right. \\
& \left.-\frac{\sqrt{\pi}}{12} \frac{e^{D}}{\left(1-\frac{1}{g} e^{D}\right)^{2}}(\nabla \zeta \cdot \nabla D)^{3}-\frac{1}{384 \pi} e^{-2 D}\left[1-\frac{1}{g} e^{D}\right]^{4}\left[(\nabla D)^{2}-4\right]\right\}
\end{aligned}
$$

Note that $\zeta$ has only derivative couplings and therefore the action (9) is invariant under a shift symmetry $\zeta \rightarrow \zeta+$ const. Since space-time is flat in this case we ignore curvature terms in the action. Higher derivative terms like $(\nabla D \cdot \nabla D)^{2}$ cannot be ruled out at this point. In what follows we treat the above Lagrangian as if it were the exact Lagrangian. It is straight forward to verify that indeed the field configurations in Eq.(8) is an exact solution of the equations of motion.

It is important to note that all interaction terms in (9) are proportional to $g(D)=$ $4 \sqrt{\pi} \frac{e^{D}}{\left(1-\frac{1}{g} e^{D}\right)^{2}}$ and, therefore $g(D)$ is the effective coupling parameter of the theory. The general solution of the equations of motion is given by

$$
<G_{\mu \nu}>=\eta_{\mu \nu}, \quad<D>=a(t-\bar{t})+b(\tau-\bar{\tau}), \quad<\zeta>=\frac{1}{g}+c
$$

where $a, b, c, \bar{t}$ and $\bar{\tau}$ are real parameters, $b^{2}-a^{2}=4$ and $c, \bar{t}, \bar{\tau}$ are arbitrary. 
There is an interesting vacuum structure which is the combination of two solutions. That is, take $\langle\zeta\rangle=\frac{1}{g}$ and $\langle D\rangle=-2 \tau$ for $\tau \geq \tau_{0}^{I}=\ln \sqrt{\frac{1}{g}}$, henceforth called region $I$, and $<D>=-2\left(\tau-2 \ln \sqrt{\frac{1}{g}}+\pi\right)$ for $\tau \leq \tau_{0}^{I I I}=\ln \sqrt{\frac{1}{g}}-\pi$, henceforth called region III. The spatial interval $\ln \sqrt{\frac{1}{g}}-\pi \leq \tau \leq \ln \sqrt{\frac{1}{g}}$ is called region $I I$. We plot these regions in Figure 1.

The physical interpretation of this vacuum state is the following. In regions $I$ and $I I I$, away from the boundary points, the effective coupling parameter is small and physics is described by the effective field theory (9). As $\tau$ approaches $\ln \sqrt{\frac{1}{g}}$ from the right and as $\tau$ approaches $\ln \sqrt{\frac{1}{g}}-\pi$ from the left, the coupling parameter blows up and a region of strong coupling is encountered. Region II is terra incognita. Perhaps new, previously unknown, dynamics applies there.

If all we knew was the effective field theory, then we would have no interpretation of physics in region $I I$. However, comparing the vacuum of Figure 1 to the matrix model solution, we know exactly how to describe physics in region II. Physics in that region is described by the low density collective field theory.

The action for Euclidean space-time effective field theory is easily obtained from Eq.(9) by analytic continuation of the time variable $t$ to Euclidean time $\theta$. We do not write it down explicitly. The effective coupling parameter and the static solutions of the Euclidean equation of motion are still given by the Euclidean continuation of the same expressions as before. It follows that in Euclidean space, the vacuum structure Figure 1 is valid. Region $I I$ is now described by the analytic continuation of the low density collective field theory to Euclidean space. There is now a non-trivial excitation of one eigenvalue in region $I I$ that connects the vacua of region $I$ and region $I I I$. This single eigenvalue excitation in Euclidean space was constructed previously, and presented in terms of the collective field theory in Eq.(四). Rewriting this solution in the $\tau$ coordinate, we find

$$
\phi_{\text {inst }}(\tau, \theta)=\frac{1}{\sin \left(\theta-\theta_{0}\right)} \delta\left(\tau-\left[\ln \sqrt{\frac{1}{g}}-\left(\theta-\theta_{0}\right)\right]\right)
$$

This is an instanton which corresponds to the tunneling of a single eigenvalue across the barrier from $\tau=\ln \sqrt{\frac{1}{g}}$ at $\theta=\theta_{0}$ to $\tau=\ln \sqrt{\frac{1}{g}}-\pi$ at $\theta=\theta_{0}+\pi$. Note that the velocity of the eigenvalue at either side of the barrier vanishes. Therefore, the Euclidean conjugate momentum of the instanton in region $I I$, matches continuously at the boundaries with the vanishing conjugate momentum of the static vacua $\phi_{0}$ in regions $I$ and $I I I$. We represent this tunneling process in Figure 1. 
Figure 1. Instanton Connecting regions $I$ and $I I I$.

In terms of $\zeta$ the instanton is given by $\zeta_{\text {inst }}(\tau, \theta)=\Theta\left(\tau-\left[\ln \frac{1}{\sqrt{g}}-\left(\theta-\theta_{0}\right)\right]\right)$. Therefore, the instanton field configuration is simply a kink moving in Euclidean time. The position of the kink is where the argument of the $\Theta$ function vanishes.

We want to stress that this configuration is not a solution of the $D, \zeta$ effective field theory. We have not included explicitly the terms in the field theory that correspond to self energy subtraction. However once they are taken into account, the instanton configuration has a finite action $\frac{\pi}{g}$, as shown in Eq.(5). Finally, we note that the initial tunneling time of the instanton, $\theta_{0}$ is arbitrary.

We integrate over the instantons and represent their effects as effective terms in the $D, \zeta$ theory. Since $\zeta$ is the light field we restrict our attention to $\zeta$ operators. The effective operators are especially important. They provide the bridge between the low density, discrete, sector of the theory and the continuous sector. A more detailed analysis is given in [9].

The instanton has three parameters, $\bar{\tau}, \theta_{0}$ and $\alpha$. The parameters $\bar{\tau}$ and $\theta_{0}$ were defined in Eqs.(10) and (11). The parameter $\alpha$ is related to the parameter $a$ in the Euclidean space continuation of Eq.(10), $a=2 \sin \alpha$. Changing $\alpha$ results in the rotation of the vacuum solution in $\tau-\theta$ space. There are three zero modes corresponding to the three broken generators of the Euclidean group associated with $\bar{\tau}, \theta_{0}, \alpha$. These have to be integrated and produce a volume factor $V o l \propto \int d \bar{\tau} d \theta_{0} d \alpha$. 
The dilute gas summation over instantons induces effective terms in the $D, \zeta$ Lagrangian. The most general action induced by instantons is $\Delta S=\int d \tau d \theta\left\{\sum_{n} C_{n} O_{n}(\tau, \theta)\right\}$, where $O_{n}$ are local operators built from $D$ and $\zeta$ and their derivatives. The coefficients $C_{n}$ can be computed by expanding the action around the instanton background. All the coefficients $C_{n}$ are proportional to the universal factor of the exponent of the instanton action and the remaining factor depends on the particular operator that is considered. Since the "size" of the instanton is $\sqrt{g}$, the dimension of the operator determines the $g$ dependence of $C_{n}$. That is

$$
C_{n}=\tilde{C}_{n} g^{d(n)} e^{-\frac{\pi}{g}}
$$

where $d(n)=\left[\operatorname{dimension}\left(O_{n}\right)\right]^{\frac{1}{2}}-1$ and $\tilde{C}_{n}$ is a numerical coefficient. The coefficient $\tilde{C}_{n}$ is not expected to be particularly large or particularly small.

We are interested in large $\frac{1}{g}$ that corresponds to small $g$. In that case the dominant and most interesting operator is the unit operator. All other operators are suppressed by powers of $g$. The coefficient of the unit operator is given by $C_{0}=\tilde{C}_{0} \frac{1}{g} e^{-\frac{\pi}{g}}$. This result was obtained in the background of a constant field $\langle\zeta\rangle=\frac{1}{g}$. Lorentz invariance then dictates that at least for slowly varying fields the effective operator depends on the full field $\zeta$ and not just its constant mode $\frac{1}{g}$. Therefore the final result for the induced operator is

$$
\Delta \mathcal{L}_{0}=\tilde{C}_{0} \zeta e^{-\pi \zeta}
$$

This operator breaks the $\zeta$ shift symmetry. It induces a runaway non-perturbative potential for the field $\zeta$.

Let us now discuss the relation of the effective field theory (9) to string theory. The class of $1+1$ dimensional string theories that we are interested in is described by the following two dimensional $\sigma$-model [14],

$$
I=\frac{1}{4 \pi} \int d^{2} z \sqrt{\hat{g}}\left\{\hat{g}^{\alpha \beta} G_{\mu \nu} \partial_{\alpha} X^{\mu} \partial_{\beta} X^{\nu}+\hat{R} D(X)+2 T(X)\right\}
$$

where $\hat{g}_{\alpha \beta}$ is the fixed world sheet metric with Euclidean signature and $\hat{R}$ is the corresponding Ricci scalar. The sigma model field $X_{\mu}$ stands for two scalar fields, $X_{0}(z)$, and $X_{1}(z)$. The field $G_{\mu \nu}(X)$ is the target space metric, assumed here to have Euclidean signature, $D(X)$ is the dilaton, and $T(X)$ is the tachyon.

Consistent string backgrounds are described by conformal field theories. The conditions for conformal invariance are determined in general by the equations $\beta=0$. The lowest order equations for the theory described by Eq.(14) are

$$
R_{\mu \nu}+2 \nabla_{\mu} \nabla_{\nu} D=0, \quad-\frac{1}{2} \nabla^{2} D+(\nabla D)^{2}+4=0, \quad-\nabla^{2} T+2 \nabla D \cdot \nabla T-4 T=0
$$


We can compare Eqs. (15) to the lowest order (in $g(D)$ ) equations of motion derived from (9). From this comparison we deduce that, to this order, the field $D$ appearing in (9) is the same as the dilaton $D$ in (14) and that the field $\zeta$ in (9) is related to the tachyon, $\zeta \propto T e^{-D}$. This result that we obtained is rather remarkable. It says that the equations of motion of the background fields for the class of string theories specified by the action (14) are, to lowest order, identical to the equations of motion of the effective field theory extension of matrix models. Can we extend this identification beyond lowest order? To do this, let us consider the solutions of the equations of motion (15). The flat space solutions are $G_{\mu \nu}=\delta_{\mu \nu}, D=a\left(X_{0}-\bar{X}_{0}\right)+b\left(X_{1}-\bar{X}_{1}\right), T=m e^{D}$, where $a^{2}+b^{2}=4$. Of particular interest is the static solution $G_{\mu \nu}=\delta_{\mu \nu}, D=-2 X_{1}, T=m e^{-2 X_{1}}$. By substituting this static solution into the sigma model (14) and writing $X_{1}=\varphi$, the following world sheet conformal field theory is obtained

$$
I=\frac{1}{4 \pi} \int d^{2} z \sqrt{\hat{g}}\left\{\hat{g}^{\alpha \beta} \partial_{\alpha} X_{0} \partial_{\beta} X_{0}+\hat{g}^{\alpha \beta} \partial_{\alpha} \varphi \partial_{\beta} \varphi-2 \hat{R} \varphi+2 m e^{-2 \varphi}\right\}
$$

which can be identified as the Liouville conformal field theory with $c_{m}=1$ matter. To obtain a Minkowski signature string theory one has to analytically continue $X_{0} \rightarrow i t(z)$, which then becomes the time variable of target space. The field $\varphi$ corresponds to the spatial dimension of target space.

Liouville theory (16) was compared to matrix models and to collective field theory. Specifically, the complete theory, beyond the lowest order was studied. The conclusion is that they describe of the same theory. Evidence to this effect was obtained on many levels e.g., see [15]-20]. For a review and more comprehensive list of references see ref. [19. In particular, the relation between $\zeta$ and $T$ as well as the linear relation between $\varphi$ and $\tau$ have been well documented.

More important, from our point of view, is that, by computing scattering amplitudes of fluctuations around the Liouville vacuum, one can determine the equations of the original string backgrounds $D$ and $T$ beyond the lowest order. When these are compared to the full equations of motion derived from Eq.(9), one finds that they are identical, as long as the two $D$ fields are identified, $\zeta=T e^{-D}$ holds and the parameter $m$ in is chosen to be $m=\frac{1}{g}$. We conclude, therefore, that the string theories associated with the world sheet action (14) have the same equations of motion and effective action for their background fields as does the effective field theory for the matrix model given in Eq.(9). Furthermore, even the low density region of the matrix model discussed earlier is expected to to describe physical aspects of these string theories, such as their non-perturbative behaviour. It follows that 
the discussion of vacua, single eigenvalue instantons and induced operators given in the previous section is, in fact, applicable to the string theories associated with (14). The instanton in Eq.(11) is therefore a "stringy" instanton.

It is tempting to conjecture that stringy instantons similar to our stringy instantons appear in 4 dimensional superstring theories and that they induce non-perturbative operators of the type discussed previously. In that case these operators are expected to be proportional to the universal factor $e^{-\sqrt{S}}$, where $S$ is a complex field that naturally appears in the effective low energy supergravity field theory obtained from superstring theory. The dilaton is related to the real part of $S,<\operatorname{Re} S>\sim \frac{1}{g^{2}}$. Note that the non-perturbative effects considered previously in the literature induced operators that were proportional to the universal factor $e^{-S}$. Since the coupling parameter $g$ is expected to be small, the difference between these two universal factors is quite big. This may have important phenomenological consequences.

ACKNOWLEDGEMENT: It is a pleasure to thank Lee Brekke, Joanne Cohn, Rick Davis, Antal Jevicki and especially Shanta De Alwis for useful discussions. This work was supported in part by the Department of Energy under contract No. DOE-AC02-76-ERO-3071. 


\section{References}

[1] D. J. Gross, N. Miljkovic, Phys.Lett.B238 (1990) 217; P. Ginsparg and J. Zinn-Justin, Phys. Lett. B240 (1990) 333; E. Brezin, V. Kazakov and Al. B. Zamolodchikov, Nucl. Phys. B338 (1990) 673.

[2] S.R. Das and A. Jevicki, Mod. Phys. Lett. A5 (1990) 1639.

[3] J. Polchinski, Nucl.Phys. B346 (1990) 253.

[4] S. Shenker, Cargese Workshop on Random Surfaces, Quantum Gravity and Strings, Cargese, France (1990).

[5] P. Ginsparg and J. Zinn-Justin, Phys. Lett. B255 (1991) 189.

[6] O. Lechtenfeld, Int. J. Mod.Phys. A7 (1992).

[7] A. Dabholkar, Nucl.Phys. B368 (1992) 293.

[8] J. D. Cohn and H. Dykstra, Mod.Phys.Lett. A7 (1992) 1163.

[9] R. Brustein and B. Ovrut, Penn preprint, UPR-523T (1992).

[10] B. Sakita, Quantum Theory of Many-Variable Systems and Fields, World Scientific, Singapore 1985.

[11] J. D. Cohn and S.P. De Alwis, Nucl.Phys.B368 (1992) 79.

[12] D. Karabali and B. Sakita, Int. J. Mod. Phys. A6 (1991) 5079.

[13] R. Brustein and S. De Alwis, Phys. Lett. B272 (1992) 285.

[14] S. Elitzur, A. Forge and E. Rabinovici, Nucl. Phys. B359 (1991) 581;

A. Tseytlin, Phys. Lett. B264 (1991) 311.

[15] D. J. Gross and I.R. Klebanov, Nucl.Phys. B352 (1991) 671.

[16] D. J. Gross and I. R. Klebanov, Nucl. Phys. B359 (1991) 3.

[17] G. Moore, Nucl.Phys. B368 (1992) 557.

[18] K. Demeterfi, A. Jevicki and J. P. Rodrigues, Nucl.Phys. B362 (1991) 173 and Nucl.Phys. B365 (1991) 499.

[19] K. Demeterfi, A. Jevicki and J. P. Rodrigues, Mod. Phys. Lett. A6 (1991) 3199.

[20] P. Di Francesco, D. Kutasov, Nucl. Phys. B375 (1992), 119. 